\title{
Local Extensions in Singular Space-Times II
}

\author{
C. J. S. Clarke \\ Department of Mathematics, University of York, Heslington, York YOl 5DD, England
}

Abstract. Previous results of the author are corrected by reformulating them in space-times whose Riemann tensor satisfies a Hölder condition.

\section{Introduction}

In an earlier paper with this title [2] I showed the existence of local extensions through quasiregular singularities (in the terminology of [5]) by (implicitly) assuming that a spacetime with a $C^{k-2}$ Riemann tensor had a $C^{k}$ metric. This assumption may not be correct (the alleged proof which I gave in [3] being invalid). The basic results do hold, however, if one uses $C^{k, \alpha}$ conditions (a Hölder condition with exponent $\alpha, 0<\alpha<1$, on the $k^{\text {th }}$ derivative). The technical tools needed to modify the proof are given in detail in [4]; the aim of the present paper is to outline their application to local extensions.

We first clarify the term "local extension" of a spacetime $(M, g)$, of which there are two definitions in the literature. Here, and in [5], it means an isometry $\phi: U \rightarrow M^{\prime}$, where $U \subset M$ and $\left(M^{\prime}, g^{\prime}\right)$ is a spacetime, such that

(i) $U$ contains a curve $\gamma$ which is incomplete with respect to a generalised affine parameter and inextendible in $M$.

(ii) $\phi \circ \gamma$ is extendible in $M^{\prime}$.

Hawking and Ellis [6], on the other hand, replace (i) by the condition that $\bar{U}$ is not compact in $M$, and (ii) by the condition that $\phi(U)$ is compact in $M^{\prime}$. This has the undesirable consequence that Minkowski space is locally extendible [1]. With the author's definition, certain compact space-times having trapped geodesics may be locally extendible.

\section{Results}

The theorem will be formulated for the case where $\gamma$ in the definition above is a broken geodesic. Since any rectifiable curve can be approximated by a broken geodesic this is no loss of generality, and it enables us to give a concrete description of the set $U$ that can be extended. In addition we impose a restriction ((iv) below) that corresponds to the non-spiral condition imposed in the earlier paper [2]. The theorem will only be proved for $C^{0, \alpha}$ Riemann tensors; but it is clear that the procedure extends to $C^{k, \alpha}$. 
Theorem. Let $\gamma:(0,1] \rightarrow(M, g)$ be an incomplete curve such that $\gamma \mid\left(t_{i}, t_{i-1}\right)$ is a geodesic for some sequence $0<\ldots<t_{n}<\ldots<t_{1}<t_{0}=1$. Let there be given a sequence $\left(a_{n}\right)_{n \in \mathbb{N}}$ and a frame $(E)_{i=0}^{3}$ (not necessarily orthonormal) parallely propagated on $\gamma$ such that the following are satisfied:

(i) $a_{n} \rightarrow 0(n \rightarrow \infty)$.

(ii) The map $T_{n}: \mathbb{R}^{4} \ni \xi \mapsto \exp \left(\xi^{i} E_{i}\left(t_{n}\right)\right)$ is defined and $1-1$ in the ball $B_{n}^{r}:=$ $\left\{\xi \mid\|\xi\|<r a_{n}\right\}$ for all $r \leqq 1$, and its image contains both $\gamma\left(t_{n-1}\right)$ and $\gamma\left(t_{n+1}\right)$.

(iii) There is a bounded (with respect to the b-metric [6]) section of the frame bundle over the set $U:=\bigcup_{n} T_{n}\left(B_{n}^{1 / 2}\right)$, the section containing $(\underset{i}{E})$, on which the components $R_{n p q}^{m}$ of the Riemann tensor satisfy a uniform Hölder condition with exponent $\alpha$.

(iv) There is a constant $K>0$ such that $\dot{\gamma}^{0}(s)>K$ (where $\left.\dot{\gamma}=\dot{\gamma}^{i} E\right)$ for all $s \in(0,1]$.

Then there is a local extension $\phi: U \rightarrow M^{\prime}$, where $\left(M^{\prime}, g^{\prime}\right)$ is a spacetime whose Riemann tensor satisfies a Hölder condition with exponent $\alpha$.

Proof. (In the following we merely outline certain operations that have been fully described in [4]). Because of (i) and (iii) (the latter implying continuity of $R_{n p q}^{m}$ ), for large enough $N$ the dimensionless quantity $a_{n}\|R\|^{1 / 2}(n>N)$ is so small that no geodesics constructed in $T_{n}\left(B_{m}^{\prime}\right)$ will focus or intersect (cf. $\S \S 3.2,3.3$ of [4]). We define $U_{n}=\bigcup_{i=N}^{n} T_{i}\left(B_{i}^{1 / 2}\right)$ and proceed by induction on $n$ : we assume $U_{n}$ to be extended; then append $T_{n+1}\left(B_{n+1}^{1 / 2}\right)$; and finally let $n \rightarrow \infty$.

Suppose, then, that we have a map $\phi_{n}: U_{n} \rightarrow M^{\prime}=\mathbb{R}^{4}$ which is an isometry for a metric $g_{(n)}$ on $M^{\prime}$. The extension of this to $\phi_{n+1}$ is performed by using a special coordinate system on $T_{n+1}\left(B_{n+1}^{1 / 2}\right)$, as follows. Choose four independent vectors $\underset{0}{Z}, \ldots, Z \in \mathbb{R}^{4}$ which are such that each of $Z_{i}^{a} E$ (for $i=0, \ldots, 3$ ) is future-pointing and timelike.

Define

$$
\begin{aligned}
P_{i} & :=\exp \left(a_{n+1} Z_{i}^{a} \underset{a}{E}\left(t_{n+1}\right)\right), \\
P_{i}^{\prime} & =\exp \left(-a_{n+1} Z_{i}^{a} \underset{a}{E}\left(t_{n+1}\right)\right), \\
S_{i} & :=\left\{x \in T_{n+1}\left(B_{n}^{\prime}\right) \mid d\left(P_{i}, x\right)=d\left(P_{i}^{\prime}, x\right)\right\}, \\
z^{i}(x) & :=d\left(x, S_{i}\right)+\int_{1}^{t_{n+1}} g_{a b} \ddot{\gamma}^{a} z_{i}^{b} \mathrm{~d} s .
\end{aligned}
$$

Here $d$ denotes the supremum of the geodesic distance for timelike geodesics, the quantities only being defined where this is finite, and $g_{a b}$ are the components of the metric in the frame $(E)$.

The functions $z^{i}$ are the required coordinates. They are constructed to achieve the following two properties:

(a) The Hölder constants of components of the metric, and their first derivatives, are bounded in terms of the Hölder constant of the Riemann tensor.

(b) Coordinates defined from adjacent $n$-values (i.e. in $T_{n}\left(B_{n}^{1 / 2}\right)$ and $T_{n+1}\left(B_{n+1}^{1 / 2}\right)$ ) approximately agree in the overlap of their domains. 
The verification of (a) is very similar to the proof of 6.1 in [4]. We let $\stackrel{i}{V}$ denote the tangent vector at $x$ to the geodesic from $x$ to $S_{i}$ that minimises the distance, with proper-time parametrisation. Then it can be shown that $g^{i j}=g(\stackrel{i}{V}, \stackrel{j}{V})$ and that

$$
g^{i j}{ }_{k}=g_{k m}\left(g\left(\nabla_{V} \stackrel{i}{V}, \stackrel{j}{V}\right)+g\left(\stackrel{i}{V}, \nabla_{V} \stackrel{j}{V}\right)\right) \text {. }
$$

The quantity $\nabla_{V}^{m} \stackrel{i}{V}$ can then be computed from the Riemann tensor, using Jacobi's equation for the variation in the geodesic defining $\stackrel{i}{V}$.

Calculations for (b) are similar, though a bit more involved. We replace the " $t_{n+1}$ " in the definitions of $z^{i}, P_{i}$ and $P_{i}^{\prime}$ by a variable parameter $s$, and vary $s$ from $t_{n+1}$ to $t_{n}$. As $s$ varies, $P_{i}$ and $P_{i}^{\prime}$ describe curves, whose tangent vectors are related to the tangent vector on $\gamma$ by Jacobi's equation. These variations in turn give rise to changes in the vector field $\stackrel{i}{V}$, from which the variation of $Z^{i}$ as $s$ is varied can be calculated: it turns out to involve only integrals of the Riemann tensor.

In this way we can construct coordinates $z^{i}$ in $T_{n+1}\left(B_{n+1}^{1 / 2}\right)$, and related coordinates - $z^{i^{\prime}}$, say - in $M^{\prime}$, using $\phi_{n *} \underset{a}{E}\left(t_{n+1}\right)$ instead of $\underset{a}{E}\left(t_{n+1}\right)$. A map $\bar{\phi}_{n+1}: T_{n+1}\left(B_{n+1}^{1 / 2}\right) \rightarrow M^{\prime}$ is then defined by relating points with equal $z^{i}$ and $z^{i \prime}$ coordinates.

The extended map $\phi_{n+1}$ is then defined by "patching" smoothly together $\phi_{n}$ and $\bar{\phi}_{n+1}$. Condition (iv) ensures that the image of $\bar{\phi}_{n+1}$ overlaps at most a fixed finite number of the previously constructed coordinate domains, and in these property (b) of the coordinates ensures that the Hölder constants of $g_{i j, k}$ and the Riemann tensor for the metric $\left(\phi_{n+1}^{*}\right)^{-1} g$ is of the order of magnitude of that of the Riemann tensor, measured in the frame bundle.

We now have a map $\phi_{n+1}$ on $U_{n+1}$, inducing a metric on its image whose Hölder constants are known. The metric $g_{(n+1)}$ is then defined by extending the difference between the induced connections $\left(\Gamma_{(n)}\right.$ and $\left.\Gamma_{(n+1)}\right)$ from $\phi_{n+1}\left(U_{n+1}\right)$ to the whole of $M^{\prime}$, followed by a redefinition of the metric exactly as in $[4], \S 8.3$. It is here that the use of Hölder conditions, rather than mere continuity, is essential.

Convergence of $g_{(n) i j, k}$ and $R_{(n) j k l}^{i}$ is then easily verified, with the Hölder conditions being respected.

\section{References}

1. Beem, J. K.: Commun. Math. Phys. 72, 273 (1980)

2. Clarke, C. J. S.: Commun. Math. Phys. 32, 205 (1973)

3. Clarke, C. J. S.: Math. Proc. Camb. Phils. Soc., 81, 279 (1977)

4. Clarke, C. J. S.: Space-times of low differentiability and singularities. J. Math. Anal. Appl. (to appear)

5. Ellis, G. F. R., Schmidt, B. G.: Gen. Rel. Grav. 8, 915 (1977)

6. Hawking, S. W., Ellis, G. F. R.: The large scale structure of spacetime. Cambridge: Cambridge University Press 1973 
